\title{
钾代谢相关基因在烟草中的表达
}

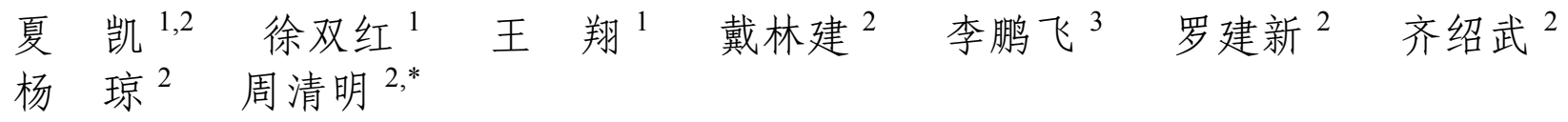

${ }^{1}$ 湖南中烟工业有限责任公司, 湖南长沙 $410014 ;^{2}$ 湖南农业大学, 湖南长沙 $410128 ;{ }^{3}$ 云南省烟草公司文山州公司, 云南文山 663000

摘 要: 以钾高效基因型 $\mathrm{K} 2 、 \mathrm{~K} 3 、 \mathrm{~K} 5 、 \mathrm{~K} 7 、 \mathrm{~K} 9$ 与常规烤烟品种 $\mathrm{K} 326$ 为材料, 采用漂浮育苗、移栽沙培的方法, 利 用实时定量 PCR 调查钾代谢相关基因在烟草叶片中的表达情况, 并测定不同材料的钾吸收动力学参数和钾利用率。 结果表明, $\mathrm{K} 2 、 \mathrm{~K} 7$ 和 $\mathrm{K} 9$ 是典型的富钾型, 12 个钾代谢相关基因中 TORK1 和 NtTPK1 的表达水平相对较高。5 个富 钾型的钾吸收能力显著强于 $K 326$ 。但它们在高钾环境下钾素吸收能力强而利用率较低, 在低钾环境下钾吸收能力强 且利用率相对较高, 尤其是品系 K2、K7 和 K9 钾的经济利用率相对较高。

关键词：钾代谢；基因；表达；烟草

\section{Expression of Potassium Metabolism-Related Gene in Tobacco}

XIA Kai ${ }^{1,2}$, XU Shuang-Hong ${ }^{1}$, WANG Xiang ${ }^{1}$, DAI Lin-Jian ${ }^{2}$, LI Peng-Fei ${ }^{3}$, LUO Jian-Xin ${ }^{2}$, QI Shao-Wu ${ }^{2}$, YANG Qiong ${ }^{2}$, and ZHOU Qing-Ming ${ }^{2, *}$ ${ }^{1}$ Hunan Tobacco Industrial Co., Ltd, Changsha 410014, China; ${ }^{2}$ Hunan Agricultural University, Changsha 410128 , China; ${ }^{3}$ Wenshan Company of
Yunnan Provincial Tobacco Company, Wenshan 663000, China

Abstract: The quality of flue-cured tobacco in production is currently limited by low potassium level in leaf. A simple and effective way to alleviate the supply of potassium in soil is screening the potassium-enriched flue-cured tobacco types. In the experiment, the tobacco seedlings were cultured in nutrient solution, and then replanted in sand soil. To investigate the potassium metabolism related gene's expression and kinetic parameters of potassium uptake and utilization with six different lines including five potassium-enriched genotypes of K2, K3, K5, K7, and K9 and a conventional genotype of K326. The results showed that the tobacco lines $\mathrm{K} 2, \mathrm{~K} 7$, and $\mathrm{K} 9$ were typical potassium-enriched genotypes, TORK1 and NtTPK1 out of 12 genes performed high expression. The ability of potassium absorption of K3, K5, K9, K7, and K2 was significantly stronger than that of K326. The potassium absorption abilities of potassium-enriched types of $\mathrm{K} 9, \mathrm{~K} 2, \mathrm{~K} 7, \mathrm{~K} 3$, and $\mathrm{K} 5$ were stronger but their use efficiencies were lower than these of K326 in high potassium environment, and higher in low potassium environment, especially for the genotypes K2, K7, and K9.

Keywords: Potassium metabolism; Gene; Expression; Nicotiana tabacum

含钾量是优质烟叶最为重要的品质指标之一，烟叶 中的钾离子不但参与烟叶生理生化反应, 与烟株的抗逆 性有密切关系, 同时还决定着烟草内在品质和工业可用 性 ${ }^{[1]}$ 。对卷烟企业来说, 烟叶含钾量是评价烟叶品质的重 要指标之一, 同时也与烟叶的安全性有较强的相关性 ${ }^{[2]}$ 。 在我国, 大部分烟区烤烟的含钾量仅为 $1.5 \%$ 左右, 含钾 量较高的也只有 $2 \%$ 左右, 与美国等先进国家有较大的差 距 ${ }^{[3]}$ 。钾是植物生长必需的大量营养元素, 有重要的营养 和生理作用, 同一植物不同品种或不同植物对钾的吸收、 分配、转运和利用效率等方面存在着很大的差异。在低钾 条件下, 高效基因型与低效基因型相比, 具有明显的生
长优势。钾效率的高低归结于吸收效率和利用效率两个方 面, 即根系对土壤钾的活化和吸收能力; 以及植物体内 钾的运输、同化等利用能力。如何提高烟叶的含钾量是当 前烟草工业研究的重点与难点。烟叶含钾量取决于烟草品 种基因型和在环境中的表达。因此, 选择一定施钾量下吸 收和积累能力强的品种类型无疑是最有效的措施, 而当 前持续依靠增施钾肥以提高烟叶含钾量的方法无法治本。

植物细胞通过其膜上的各种 $\mathrm{K}^{+}$通道和转运体蛋白转 运 $\mathrm{K}^{+}$进出细胞, 这些通道和转运体的分子调节机制迄今 仍不十分清楚, 但已有部分相关基因得到克隆和研究。植 物中第一个鉴定的 $\mathrm{K}^{+}$运输的基因是 $K A T 1^{[4-5]}$, 互补试验

\footnotetext{
本研究由湖南中烟工业有限公司项目 (2008-YC-0001) 资助。

* 通讯作者(Corresponding author): 周清明, E-mail: zqm8051@hunau.net, Tel: 0731-84618051

第一作者联系方式: E-mail: xiak0502@hngytobacco.com, Tel: 0731-85559462

Received(收稿日期): 2011-09-21; Accepted(接受日期): 2012-01-15; Published online(网络出版日期): 2012-04-06.

URL: http://www.cnki.net/kcms/detail/11.1809.S.20120406.0948.007.html
} 
证明 $K A T 1$ 基因可恢复酵母突变体在低钾水平下的生长。 烟草的 NtTPK 1 基因编码一个疏水膜蛋白, 包含典型的 $\mathrm{K}^{+}$ 吸收转运功能保守域, 并且与主要的高等植物 $\mathrm{K}^{+}$吸收转 运基因具有较高的同源性 ${ }^{[6]}$ 。NtTPK1 具有 TXXTXGYGD 的特征序列, 因此, 可能具有 $K C O$ 家族的 $\mathrm{K}^{+}$吸收与转运 的生物学功能。另外烟草 $\mathrm{K}^{+}$通道基因 $N K T 1^{[7]}$ 的主要功能 是吸收 $\mathrm{K}^{+} ; G O R K$ 在保卫细胞中表达, 控制 $\mathrm{K}^{+}$流入和流出 保卫细胞 ${ }^{[8-9]}$ 。CIPK23通过磷酸化作用调控 $\mathrm{K}^{+}$通道蛋白 $K A T 1$ 的活性, 从而调节植物在低 $\mathrm{K}^{+}$胁迫下的 $\mathrm{K}^{+}$吸收和转 运, 而 $C I P K 23$ 的活化又依赖于钙信号感受器 $C B L 1$ (或 $C B L 9)$ 在质膜上的正向调控 ${ }^{[10]}$ 。研究 $K A T 1 、 N t T P K 1$ 和 $N K T 1$ 等基因的表达调节与 $\mathrm{K}^{+}$吸收和转运关系, 了解调节 $\mathrm{K}^{+}$吸收的信号转导模型, 有助于理解 $\mathrm{K}^{+}$通道、高亲和力 $\mathrm{K}^{+}$运输体和与 $\mathrm{K}^{+}$转运有关的调控网络体系在 $\mathrm{K}^{+}$吸收和植 物矿质营养代谢中的作用。

本文通过定量实时PCR(qRT-PCR)技术, 检测高钾和 低钾不同烟草株系中与钾吸收和转运相关的 12 个基因在 叶片中的表达情况, 寻找其中与钾含量相关的关键基因, 根据这些基因的表达情况来预测选育品种的品质, 以期 为种质选育寻找快速有效的鉴定方法。

\section{1 材料与方法}

\section{1 试验材料}

应用花粉管通道法于1999年以空心莲子草、商陆和马 齿苋为供体, 烤烟品种 K326为受体, 配制导入组合 3 个, 获导入当代种子(D0) 2595 粒, 从2000-2009年, 以成熟 期分别取中部烤后烟叶测定烟叶钾含量, 以烟叶钾含量 指标高于 $2.5 \%$ 为主要选择压力, 同时考虑株型和抗病性 进行连续10年的定向选择, 篮选得到D10代 5 个株系 ${ }^{[11-12]}$, 分别为烟草钾高效基因型 ${ }^{[13-15]}$ (以下简称高钾) K2、K3、

$\mathrm{K} 5 、 \mathrm{~K} 7$ 和 $\mathrm{K} 9$, 并以烟草钾低效基因型 (以下简称低钾) $\mathrm{K} 326(\mathrm{CK})$ 作为对照, 2010年按湖南省优质烤烟生产标准 种植于湖南农业大学烟草实验基地内, 并对所有烟株进 行平顶处理。

\section{2 烟苗培养和沙培试验}

采用自然光照, 将烟草种子播种于盛有基质的漂浮盘 中育苗, 待烟苗长至5片真叶时, 选取生长均匀一致的烟苗, 于 2010 年 5 月 12 日移至内径为 $15 \mathrm{~cm}$, 高为 $20 \mathrm{~cm}$ 的塑料花盆 中沙培(沙土比为 $3 ： 1$ ), 每盆 1 株。设 6 个处理, 2 个供钾水平 (高供钾水平 $6 \mathrm{mmol} \mathrm{L}^{-1}$ 和低供钾水平 $0.6 \mathrm{mmol} \mathrm{L}^{-1}$ ), 3次重 复, 烤烟所需的钾来源于 $\mathrm{KNO}_{3}$ 。于移栽当天取各处理烟叶 提取总RNA, 采用qRT-PCR分析钾离子代谢相关基因表达 水平; 分别于移栽 $25 \mathrm{~d}$ 和移栽 $45 \mathrm{~d}$ 后, 每重复选取 5 株生长整 齐一致的烟株分析各处理钾素利用效率。

\section{3 取样和 RNA 提取}

根据Verwoerd等 ${ }^{[16]}$ 方法取K2、K3、K7、K9与对照植 株5 片真叶, 剪碎, 用液氮磨成干粉状, 立即分装至事先 装有 $1.0 \mathrm{~mL}$ Trizol提取液(Invitrogen)的 $1.5 \mathrm{~mL}$ 离心管, 每
管约 $100 \mathrm{mg}$, 标记、盖紧、摇动, 使样品与Trizol提取液充 分混合, 加 $200 \mu \mathrm{L}$ 氯仿, 振荡混匀, $4^{\circ} \mathrm{C}, 12000 \times g$ 离心 15 $\min$, 小心吸出上层水相, 转入另一离心管, 加 $500 \mu \mathrm{L}$ 异 丙醇, $-20^{\circ} \mathrm{C}$ 沉 $10 \mathrm{~min}, 12000 \times g$ 离心 $10 \mathrm{~min}$ 分离出 RNA, 再经 $75 \%$ 酒精洗涤, 室温微干后, 加适当体积RNase-free 水, 充分溶解, 测量RNA浓度。

\section{4 实时苂光定量 RT-PCR 分析基因表达特性}

使用Primer Expression 3.0 软件(Applied Biosystems, 美 国)设计实时 $\mathrm{PCR}$ 引物, 根据已知钾离子代谢相关基因 NtTPK1 (EU161633)、NKT1 (AB196790)、NtKC1 (AB 196791) $)^{[7]} 、 N K C 1$ (DQ630714) $)^{[16]} 、$ TORK1 (AB196792) ${ }^{[18]}$, CBL1 (NM_202837)、 CBL9 (NM_124081)、CIPK23 (NM_102766)、KAT1 (NM_123993)、KAT2 (NM_117939) $)^{[19]}$, GORK (NM_123109)、SKOR (NM_111153) ${ }^{[20]}$ 的cDNA全长 序列设计定量PCR引物(表1), 以烟草肌动蛋白基因(Actin gene, Accession No. U60495)为内参基因，依据其序列设 计引物(表1)。用购于Fermentas公司的DNase处理提取的 总RNA以去除混入的基因组DNA（按厂家提供的处理步 骤)。利用M-MLV反转录酶( TaKaRa)合成cDNA。以cDNA 为模板, 按照SYBR Green Realtime PCR Master Mix (Toyobo, Japan)说明书操作, 在 ABI 7900HT (Applied Biosystems，美国)上进行PCR。反应体系为 $12.5 \mu \mathrm{L}$ SYBR Green Realtime PCR Master Mix, $1 \mu \mathrm{L}$ cDNA, $10 \mu \mathrm{mol} \mathrm{L}{ }^{-1}$ $\mathrm{PCR}$ 上有引物 $0.5 \mu \mathrm{L}, 10 \mu \mathrm{mol} \mathrm{L}{ }^{-1} \mathrm{PCR}$ 下游引物 $0.5 \mu \mathrm{L}$, 补蒸 馏水至总体积 $25 \mu \mathrm{L}$ 。反应程序为 $95^{\circ} \mathrm{C} 60 \mathrm{~s} ; 35$ 循环 $\left(95^{\circ} \mathrm{C} 60\right.$ $\left.\mathrm{s}, 65^{\circ} \mathrm{C} 35 \mathrm{~s}\right)$ 。反应结束后分析苂光值变化曲线和融解曲线。 设样本和内参各 3 个重复, 采用相对定量分析法分析数据, 目的基因相对表达量 Rel. Exp $=2^{-\Delta \Delta \mathrm{Ct}}$ 。其中,$\Delta \Delta \mathrm{Ct}=$ (Calibrator $\Delta \mathrm{Ct})-$ (未知样品 $\Delta \mathrm{Ct})$, 未知样品 $\Delta \mathrm{Ct}=($ 内参基因 $\mathrm{Ct})-($ 目的基因 $\mathrm{Ct})$, Calibrator $\Delta \mathrm{Ct}=($ 参比样内参基因 $\mathrm{Ct})-($ 参 比样目的基因 $\mathrm{Ct}$ )。

\section{5 烟株根系钾吸收动力学测定}

对移栽后沙培的烟株7 d浇一次Hoagland完全营养液, 长至7 8片真叶时, 选取生长均匀一致的烟苗进行耗竭试 验。在耗竭试验开始前, 将烟苗放在无钾营养液中处理 $48 \mathrm{~h}$, 调节 $\mathrm{pH}$ 值为 6.0 , 钾饥饿处理期间每天更换饥饿液 1 次。耗竭试验开始时, 将烟苗放入含 $200 \mathrm{~mL}$ 耗竭液的黑色 三角瓶中(耗竭液的组成为: $0.4 \mathrm{mmol} \mathrm{L}{ }^{-1} \mathrm{KNO}_{3}, 0.2 \mathrm{mmol}$ $\mathrm{L}^{-1} \mathrm{CaSO}_{4}, 5 \mathrm{mmol} \mathrm{L}^{-1} \mathrm{Mes}$, 用Tris碱调 $\mathrm{pH}$ 值至6.0), 每隔 $1 \mathrm{~h}$ 取一次样, 直到耗竭液中钾浓度接近零时结束取样。 每次取 $1 \mathrm{~mL}$ 耗竭液, 同时补充 $1 \mathrm{~mL}$ 去离子水, 使耗竭液 的体积保持不变。最后在吸收液浓度保持相对稳定时取样 即可获得 $C_{\mathrm{min}}$ 值 (即吸收速率为零时环境离子的浓度), 用 火焰光度计法测定耗竭液的钾浓度。取样结束后取出植株, 用吸水纸吸干根系表面的水分, 称根鲜重。按吸收动力学 方法 ${ }^{[21]}$, 得出一元二次方程模型 $Y=a+b X+c X^{2}$ (式中, $X$ 代 表吸收时间, $Y$ 代表该时刻耗竭液中钾浓度), 获得 $a 、 b 、 c$ 值，不考虑 $a 、 b 、 c$ 的正负号。 
$I_{\max }\left(K, \mu \mathrm{mol} \mathrm{FW} \mathrm{g}{ }^{-1} \mathrm{~h}^{-1}\right)=\mathrm{b} \times$ 耗竭液体积 $(\mathrm{mL}) \times 0.06 /$ 根系鲜重 $(\mathrm{g})$
$K_{\mathrm{m}}\left(K, \mu \mathrm{mol} \mathrm{L} \mathrm{L}^{-1}\right)=a+\left[\left(V_{\max }\right)^{2}-4 b^{2}\right] / 16 c$

养分流入速率 $\alpha=I_{\max } / K_{\mathrm{m}}$

表 1 试验中采用的引物序列

Table 1 List of genes and primer sequences for real-time PCR

\begin{tabular}{lll}
\hline \multicolumn{1}{c}{ 名称 } & & $\begin{array}{c}\text { 序列 } \\
\text { Sequence }\end{array}$ \\
\hline NtTPK1 & F: 5'-GGTGTTGTGGTCCTGTGTATTGG-3' & R: 5'-AATTGAACGCTCTATCGCCATACC-3' \\
Nkt1 & F: 5'-GTAAAACGACGGCCAG-3' & R: 5'-CAGGAAACAGCTATGAC-3' \\
Ntkc1 & F: 5'-CCTCTATTGCTTCCCAATCAG-3' & R: 5'-TCTTGTTGGCTTCTGAATCC-3' \\
NKC1 & F: 5'-ATGAAGTTGGGGCAGAAAATGGTG-3' & R: 5'-AGTTCTTCCTTGGGCGTCTCC-3' \\
TORK1 & F: 5'-ACTACGAAGGCTGTTGTCCAATG-3' & R: 5'-CCAGAAGCAATCTCGCCATCG-3' \\
CBL1 & F: 5'-ATGTTTTCCACCCCAATGCTTCTC-3' & R: 5'-CAGCCAGTTTCATTTCAGATTCGC-3' \\
CBL9 & F: 5'-GCTTCCCTAGAGGAGAAAACAGAC-3' & R: 5'-CAGCCAGTTTCATTTCAGATTCGC-3' \\
CIPK23 & F: 5'-TGTTGTGGAGAGGCGAGAAGAAG-3' & R: 5'-CCGAGATTGAGACCCTGGGATG-3' \\
Kat1 & F: 5'-GGGATGGGAAGAGTGGAGAGATTC-3' & R: 5'-CATTAGAGCAGTGTCGGAAGTCG-3' \\
Kat2 & F: 5'-GAGATGTGGCTGGTCATTCTTGTG-3' & R: 5'-AGTGCGTCTTTCTTGTAGGTGATG-3' \\
GORK & F: 5'-CGCTCTATACTTCGCCATTGTCAC-3' & R: 5'-AAGCACCGAGAACCATATCAAACG-3' \\
SKOR & F: 5'-CGGCGGTAGCGGTGGAAAG-3' & R: 5'-CGGTTATCGGGATGAACAATGGAG-3' \\
Actin & F: 5'-AAGGGATGCGAGGATGGA-3' & R: 5'-CAAGGAAATCACCGCTTTGG-3' \\
\hline
\end{tabular}

1.6 不同基因型烤烟的种植及其钾含量的测定 对试验材料采用大区对比设计, 共设 4 个处理, 3 次 重复, 以 $\mathrm{K} 326$ 为对照, 每处理 $666.7 \mathrm{~m}^{2}$, 分别于 2010 年 在贵州贵阳和湖南永州两地种植, 按当地优质烤烟生产 标准管理。待各处理烟叶生长至工艺成熟期, 按三段式烘 烤工艺标准烘烤, 烤后从两地每处理取 B2F、C3F、X2F 3 个等级烟样各 $2.5 \mathrm{~kg}$, 送湖南中烟工业有限责任公司技 术中心, 分析品质。参照烟草行业标准 YC/T 173-2003, 采 用火焰光度法测定烟叶钾含量。

\section{7 数据处理}

采用 SPSS16.0 软件中 One-way ANOVA 进行方差分 析和多重比较, 用 $f$ 检验法进行显著性检验, LSD 法进行 多重比较。

\section{2 结果与分析}

2.1 钾代谢相关基因在不同品系中的表达分析 比较 NtTPK1、NKT1、NtKC1、NKC1、TORK1、CBL1、 CBL9、CIPK23、KAT1、KAT2、GORK 和 SKOR 12 个基 因在不同品系中的表达水平(图 1)。由图可知, 5 个高钾品 系的 NKC1、NKT1、CBL1、CIPK23、KAT2、SKOR 基因 表达量均明显高于对照 K326, 另外发现 K2 和 K7 这 2 个 品系中, 除 $\mathrm{K} 2$ 的 $N t k c 1$ 基因和 $\mathrm{K} 7$ 的 $N K C 1$ 基因与其他品 系差异不明显外, 所检测的其余基因表达水平要明显高 于其他品系, 因此认为这 2 个品系可能是典型的高钾材料, 另外 K9 品系中各个基因的表达水平也相对较高。

2.2 高钾基因型中各个钾代谢相关基因的表达量分析 比较 NtTPK1、NKT1、NtKC1、NKC1、TORK1、CBL1、 CBL9、CIPK23、KAT1、KAT2、GORK 和 SKOR 钾代谢
基因在 5 个典型高钾品系中的表达水平，发现基因 TORK1 和 NtTPK1 要显著高于其他基因(图 2), 另外 $C I P K 23$ 基因的表达水平也相对较高。

2.3 不同钾基因型烤烟根系钾吸收动力学参数差异

由表 2 可知, 6 个烤烟根系吸收动力学参数 $I_{\max } 、 K_{\mathrm{m}}$ 、 $C_{\text {min }} 、 \alpha$ 值都存在差异。其中 $I_{\max }$ 和 $\alpha$ 值的差异相对明显, 表现为 $\mathrm{K} 3>\mathrm{K} 5>\mathrm{K} 9>\mathrm{K} 7>\mathrm{K} 2>\mathrm{K} 326, \mathrm{~K} 3$ 根系对钾的最大吸 收速率为 $318.8 \mu \mathrm{mol} \mathrm{FW} \mathrm{g} \mathrm{F} \mathrm{h}^{-1}$, 而 $\mathrm{K} 326$ 根系对钾的最 大吸收速率为 $52.2 \mu \mathrm{mol} \mathrm{FW} \mathrm{g}{ }^{-1} \mathrm{~h}^{-1}$, 前者为后者的 6.11 倍。6 个基因型烤烟对钾的亲和力 $\left(K_{\mathrm{m}}\right)$ 和 $C_{\mathrm{min}}$ 的差异相对 较小, 表现为 $\mathrm{K} 2>\mathrm{K} 326>\mathrm{K} 7>\mathrm{K} 9>\mathrm{K} 3>\mathrm{K} 5$ 。通常 $I_{\max }$ 大, $K_{\mathrm{m}}$ 小的作物吸钾能力大、吸钾潜力高, 耐贫痊能力强, 根系 钾吸收动力学参数印证了 K3、K5、K9、K7、K2 等高钾 材料的吸钾能力强于对照 K326, 但根系钾吸收动力学参 数表明 $\mathrm{K} 7 、 \mathrm{~K} 2$ 的吸钾能力在低钾水平下弱于 $\mathrm{K} 3 、 \mathrm{~K} 5$ 、 $\mathrm{K} 9$, 这与钾代谢相关基因在 K7、K2 两个株系中表达量高 相悖, 这可能是相同烟株在不同的生育时期钾吸收能力 存在一定差异所致。

\section{4 不同钾基因型烤烟钾素利用效率分析}

由表 3 可知, 在高供钾水平下, 移栽 $25 \mathrm{~d}$ 和移栽 $45 \mathrm{~d}$ 高钾基因型烤烟的钾生物利用率明显高于对照 K 326 。在 移栽 $25 \mathrm{~d}$, 高钾基因型烤烟生物利用率的变化范围在 15.4 17.6 $\mathrm{g} \mathrm{mg}^{-1}$ 之间, 比 K326 高出 $4.6 \%$ 19.2\%; 移栽 $45 \mathrm{~d}$, 高钾基因型烤烟生物利用率的变化范围在 43.2 38.1 $\mathrm{g} \mathrm{mg}^{-1}$, 比 K326 高出 2.4\% 14.8\%。而高钾基因 型烤烟的钾经济利用率同对照 K326 间存在明显差异, 在 移栽 $25 \mathrm{~d}$, 表现为 $\mathrm{K} 2 、 \mathrm{~K} 3>\mathrm{K} 326>\mathrm{K} 5 、 \mathrm{~K} 7 、 \mathrm{~K} 9$, 但在移 栽 $45 \mathrm{~d}$ ，都低于对照 $K 326$ 。在移栽 $45 \mathrm{~d}, 5$ 个高钾基因型 

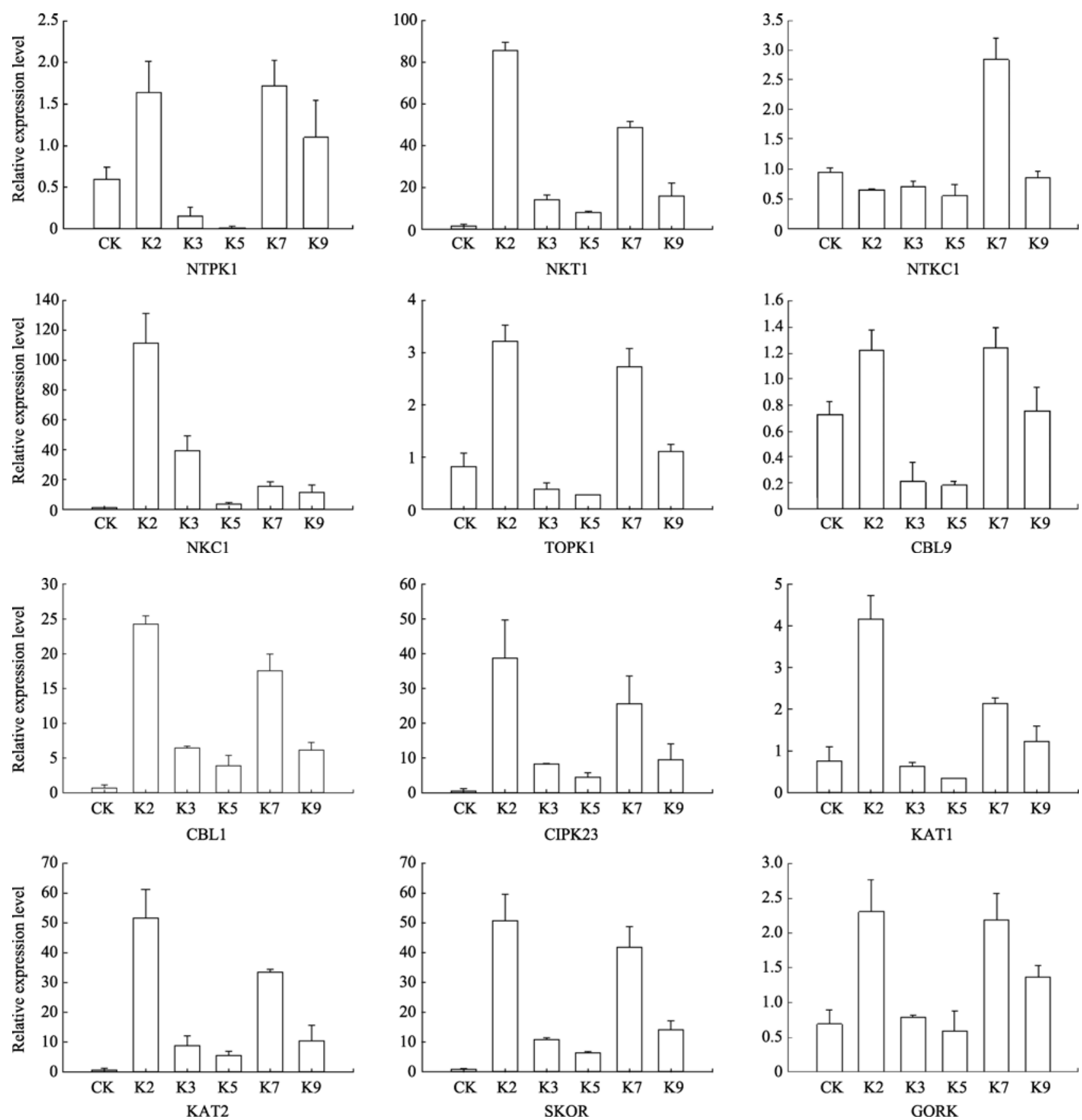

图 1 钾代谢相关基因在烟草植株中的表达

Fig. 1 Expression pattern of potassium metabolism-related genes in different tobacco plants

烤烟钾的经济利用效率较低。表明高钾基因型烤烟高钾环 境下钾素吸收能力强但利用率较低。

当外界钾浓度降为 $0.6 \mathrm{mmol} \mathrm{L}^{-1}$ 时, 移栽 $25 \mathrm{~d}$ 的高 钾基因型烤烟的钾生物利用率和经济利用率都高于 K326, 其钾生物利用率变化范围为 $68.0 \sim 98.8 \mathrm{~g} \mathrm{mg}^{-1}$, 比 K326 高 出 $18.2 \% \sim 71.9 \%$, 钾经济利用率的变化范围为 49.2 $77.4 \mathrm{~g} \mathrm{mg}^{-1}$, 比 K326 高出 $4.8 \% \sim 65.0 \%$; 在移栽 $45 \mathrm{~d}$, 各 高钾基因型烤烟(除 K3 外)的钾生物利用率高于 K326, 其 变化范围为 $170.6 \sim 211.8 \mathrm{~g} \mathrm{mg}^{-1}$, 比 K326 高出 $0.2 \%$ $24.4 \%$, 而 $\mathrm{K} 3$ 比 $\mathrm{K} 326$ 低 $0.037 \mathrm{~g} \mathrm{mg}^{-1}$ 。其经济利用率由
于生长后期烟叶重量增加不明显, K3 和 K5 分别比 K326 低 $1.28 \mathrm{~g} \mathrm{mg}^{-1}$ 和 $1.31 \mathrm{~g} \mathrm{mg}^{-1}$ 。表明高钾基因型烤烟在低 钾环境下钾素吸收能力强且利用率相对较高, 尤其是 $\mathrm{K} 2 、 \mathrm{~K} 7$ 和 K9 钾经济利用率相对较高。

2.5 不同钾基因型烤烟烟叶钾含量的比较

以 K326 为对照, 分别在贵州贵阳和湖南永州两地种 植, 分析烤后烟叶的 B2F、C3F、X2F 等级钾含量(表 4)。 结果表明, K7、K2 的上、中、下 3 个部位烟叶钾含量均 高于 $2.5 \%$, 与 K326 相比差异显著, K9 除上部叶与 K326 差异不明显外, 中部叶和下部叶与 K326 相比差异显著。 


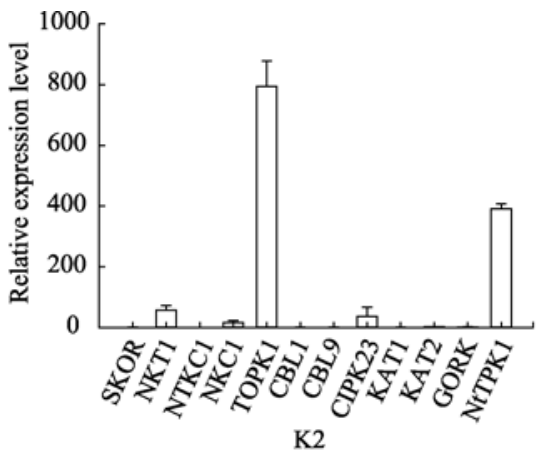

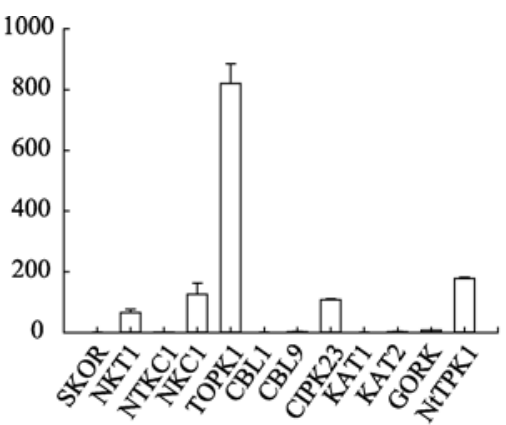

$\mathrm{K} 3$

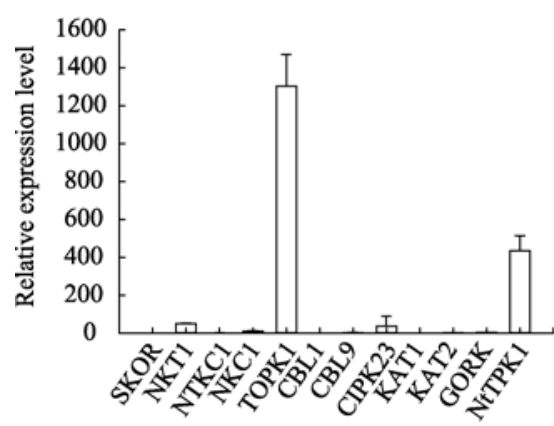

K7

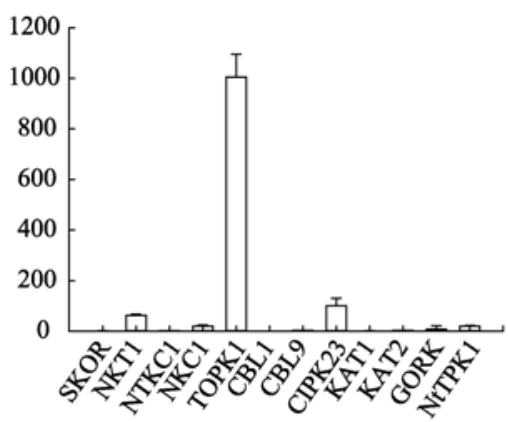

K5

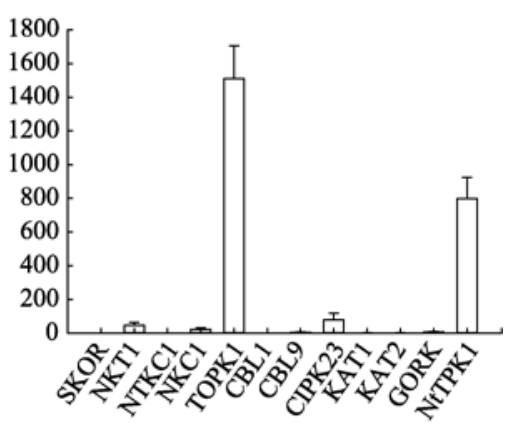

K9

图 2 钾代谢相关基因在高钾株系中的表达

Fig. 2 Expression patterns of potassium metabolism-related genes in high-K lines

表 2 不同钾基因型烤烟根系钾吸收动力学参数

Table 2 Kinetic parameters of potassium absorption in roots of different genotypes of tobacco

\begin{tabular}{|c|c|c|c|c|}
\hline $\begin{array}{l}\text { 株系 } \\
\text { Line } \\
\end{array}$ & $\begin{array}{c}\text { 最大吸收速率 } \\
I_{\max }\left(\mu \mathrm{mol} \mathrm{FW} \mathrm{g} \mathrm{g}^{-1}\right) \\
\end{array}$ & $\begin{array}{l}\text { 表观米氏常数 } \\
K_{\mathrm{m}}\left(\mu \mathrm{mol} \mathrm{L}{ }^{-1}\right) \\
\end{array}$ & $\begin{array}{l}\text { 最小临界浓度 } \\
C_{\min }\left(\mu \mathrm{mol} \mathrm{L} \mathrm{L}^{-1}\right) \\
\end{array}$ & $\begin{array}{c}\text { 养分流入速率 } \\
\alpha \\
\alpha\end{array}$ \\
\hline $\mathrm{CK}(\mathrm{K} 326)$ & $52.18 \mathrm{~d}$ & $591.95 \mathrm{~b}$ & $590.96 \mathrm{~b}$ & $0.088 \mathrm{c}$ \\
\hline $\mathrm{K} 2$ & $55.84 \mathrm{c}$ & $609.82 \mathrm{a}$ & $608.41 \mathrm{a}$ & $0.092 \mathrm{c}$ \\
\hline K3 & $318.79 \mathrm{a}$ & $551.32 \mathrm{c}$ & $537.34 \mathrm{c}$ & $0.578 \mathrm{a}$ \\
\hline K5 & $313.68 \mathrm{a}$ & $551.21 \mathrm{c}$ & $536.76 \mathrm{c}$ & $0.569 \mathrm{a}$ \\
\hline K7 & $176.86 \mathrm{~b}$ & $589.57 \mathrm{~b}$ & $585.05 \mathrm{~b}$ & $0.300 \mathrm{~b}$ \\
\hline K9 & $190.18 \mathrm{~b}$ & $588.84 \mathrm{~b}$ & $583.87 \mathrm{~b}$ & $0.323 \mathrm{~b}$ \\
\hline
\end{tabular}

同一列中标以小写字母的值在 $5 \%$ 水平差异显著。

Values within a calumn followed by different letters are significantly different at the 0.05 probability level.

$\mathrm{K} 326$ 的中部叶钾含量接近于选择压力 $2.5 \%$, 这可能与所 选试验地土壤速效钾含量有关。

\section{3 讨论}

本试验由钾代谢基因表达水平的检测和烟叶钾含量 分析的结果发现, K2、K7 和 K9 烟草品系的 TORK1 和 $N t T P K 1$ 基因的表达水平相对较高, 在植物钾含量的累积 过程中起着很重要的作用, 可能是调节 $\mathrm{K}^{+}$通道的目的基 因 $\left(\mathrm{K}^{+}\right.$in), 导致保卫细胞质膜上 HATPase 激活超极化促 使内向整流钾离子通道打开, $\mathrm{K}^{+}$内流 ${ }^{[22]}$, 或者是植物缺 钾时引起高亲和性钾转运体的诱导表达的目的基因, 这 是关键的钾饥饿胁迫响应机制 ${ }^{[23]}$ 。动力学参数排序的多 样性能反映自然界作物品种钾营养效率的多样性 ${ }^{[24]}$, 故 针对系钾吸收动力学参数对高钾株系的高效吸钾能力进 行了验证, 试验结果表明 K3、K5、K9、K7 和 K2 高钾型
的钾吸收能力强于对照 $\mathrm{K} 326$, 证明了上述品系是高效吸 钾基因型，钾营养效率是受作物本身的基因型决定的而 且是可以遗传的。但 $K 7 、 K 2$ 的吸钾能力在低钾水平下弱 于 K3、K5、K9，与前面这两个品系的基因表达量较高看 似相悖, 这可能是相同品种在生长发育的不同时期吸钾 能力有差异所致。

养分利用效率是表示植物生产力的重要指标之一, 它可以通过养分生物利用率来衡量, 而篮选植物养分高 利用率这一重要指标则可以通过养分经济利用率来衡 量 ${ }^{[25]}$ 。本文又通过测算钾素利用率对钾代谢相关基因表 达水平结果进行了验证，结果表明，高钾基因型 $\mathrm{K} 9 、 \mathrm{~K} 2$ 、 $\mathrm{K} 7 、 \mathrm{~K} 3$ 和 $\mathrm{K} 5$ 在高钾环境下钾素吸收能力强但利用率较 低, 在低钾环境下钾素吸收能力强且利用率相对较高, 尤其是 K2、K7 和 K9 株系的钾经济利用率相对较高。有 研究表明，养分利用效率高的基因型作物在养分效率较低 
表 3 各生育期不同基因型烤烟在不同供钾水平下的钾利用率

Table 3 Potassium use rate in different flue-cured tobacco genotypes under different application of potassium levels at different development stages

\begin{tabular}{|c|c|c|c|c|c|c|c|c|}
\hline \multirow[b]{2}{*}{$\begin{array}{l}\text { 株系 } \\
\text { Line }\end{array}$} & \multicolumn{4}{|c|}{ 移栽后 $25 \mathrm{~d} 25$ days after transplant } & \multicolumn{4}{|c|}{ 移栽后 $45 \mathrm{~d} 45$ days after transplant } \\
\hline & $\begin{array}{l}\text { 烟叶干物 } \\
\text { 质重 } \\
\text { Dry matter } \\
\text { weight }(\mathrm{g})\end{array}$ & $\begin{array}{c}\mathrm{K}^{+} \text {积累量 } \\
\mathrm{K}^{+} \text {accumulation } \\
(\mathrm{mg})\end{array}$ & $\begin{array}{c}\mathrm{K}^{+} \text {生物利用 } \\
\text { 效率 } \\
\text { Bioavailability } \\
\text { of } \mathrm{K}^{+}\left(\mathrm{g} \mathrm{mg}^{-1}\right)\end{array}$ & $\begin{array}{c}\mathrm{K}^{+} \text {经济利用效率 } \\
\mathrm{K}^{+} \text {economic use } \\
\text { efficiency } \\
\left(\mathrm{g} \mathrm{mg}^{-1}\right)\end{array}$ & $\begin{array}{l}\text { 烟叶干物 } \\
\text { 质重 } \\
\text { Dry matter } \\
\text { weight }(\mathrm{g})\end{array}$ & $\begin{array}{l}\mathrm{K}^{+} \text {积累量 } \\
\mathrm{K}^{+} \text {accumu- } \\
\text { lation (mg) }\end{array}$ & $\begin{array}{c}\mathrm{K}^{+} \text {生物利用效率 } \\
\text { Bioavailability of } \\
\mathrm{K}^{+}\left(\mathrm{g} \mathrm{mg}^{-1}\right)\end{array}$ & $\begin{array}{c}\mathrm{K}^{+} \text {经济利用效率 } \\
\mathrm{K}^{+} \text {economic use } \\
\text { efficiency } \\
\left(\mathrm{g} \mathrm{mg}^{-1}\right)\end{array}$ \\
\hline \multicolumn{9}{|c|}{ 处理钾浓度 Concentration of $\mathrm{K}^{+}: 6.0 \mathrm{mmol} \mathrm{L}^{-1}$} \\
\hline CK & $10.11 \mathrm{~b}$ & $804.86 \mathrm{~cd}$ & $14.76 \mathrm{~d}$ & $12.56 \mathrm{bc}$ & $26.16 \mathrm{~b}$ & $1102.88 \mathrm{~b}$ & $37.48 \mathrm{~d}$ & $23.72 \mathrm{a}$ \\
\hline $\mathrm{K} 2$ & $11.11 \mathrm{a}$ & $878.38 \mathrm{a}$ & $15.93 \mathrm{~b}$ & $12.65 \mathrm{~b}$ & $27.15 \mathrm{a}$ & $1232.11 \mathrm{a}$ & $38.40 \mathrm{~cd}$ & $22.04 \mathrm{~b}$ \\
\hline $\mathrm{K} 3$ & $11.45 \mathrm{a}$ & $823.92 \mathrm{bc}$ & $17.60 \mathrm{a}$ & $13.90 \mathrm{a}$ & $26.92 \mathrm{ab}$ & $1263.31 \mathrm{a}$ & $38.07 \mathrm{~cd}$ & $21.31 \mathrm{~b}$ \\
\hline K5 & $10.15 \mathrm{~b}$ & $842.50 \mathrm{~b}$ & $15.45 \mathrm{~cd}$ & $12.05 \mathrm{~d}$ & $26.74 \mathrm{ab}$ & $1134.05 \mathrm{~b}$ & $43.02 \mathrm{a}$ & $23.58 \mathrm{a}$ \\
\hline K7 & $9.66 \mathrm{c}$ & $779.13 \mathrm{~d}$ & $16.79 \mathrm{a}$ & $12.40 \mathrm{c}$ & $24.36 \mathrm{~d}$ & $1113.65 \mathrm{~b}$ & $40.74 \mathrm{~b}$ & $21.87 \mathrm{~b}$ \\
\hline K9 & $10.09 \mathrm{bc}$ & $842.65 b$ & $15.69 \mathrm{bc}$ & $11.97 \mathrm{~d}$ & $26.24 b$ & $1162.23 \mathrm{~b}$ & $39.87 \mathrm{bc}$ & $22.58 \mathrm{a}$ \\
\hline \multicolumn{9}{|c|}{ 处理钾浓度 Concentration of $\mathrm{K}^{+}: 0.6 \mathrm{mmol} \mathrm{L}^{-1}$} \\
\hline CK & $6.53 \mathrm{bc}$ & $139.12 \mathrm{a}$ & $57.50 \mathrm{~d}$ & $46.94 \mathrm{~d}$ & $14.12 \mathrm{c}$ & $120.28 \mathrm{~b}$ & $170.19 \mathrm{~b}$ & $117.39 \mathrm{~b}$ \\
\hline $\mathrm{K} 2$ & $7.60 \mathrm{a}$ & $98.10 \mathrm{~d}$ & $98.88 \mathrm{a}$ & $77.47 \mathrm{a}$ & $12.93 \mathrm{~d}$ & $88.10 \mathrm{c}$ & $211.80 \mathrm{a}$ & $146.77 \mathrm{a}$ \\
\hline $\mathrm{K} 3$ & $6.46 \mathrm{~cd}$ & $98.16 \mathrm{~d}$ & $86.08 \mathrm{~b}$ & $65.81 \mathrm{~b}$ & $15.06 \mathrm{c}$ & $129.70 \mathrm{~b}$ & $163.84 \mathrm{~b}$ & $116.11 \mathrm{~b}$ \\
\hline K5 & $6.81 \mathrm{bc}$ & $134.14 \mathrm{ab}$ & $67.99 \mathrm{~cd}$ & $50.77 \mathrm{~cd}$ & $17.15 \mathrm{a}$ & $147.74 \mathrm{a}$ & $170.57 \mathrm{~b}$ & $116.08 \mathrm{~b}$ \\
\hline K7 & $6.21 \mathrm{~d}$ & $126.16 \mathrm{bc}$ & $69.75 \mathrm{c}$ & $49.22 \mathrm{~cd}$ & $15.94 \mathrm{~b}$ & $115.63 \mathrm{~b}$ & $203.15 \mathrm{a}$ & $137.85 \mathrm{a}$ \\
\hline K9 & $6.94 \mathrm{~b}$ & $119.97 \mathrm{c}$ & $76.44 \mathrm{c}$ & $57.85 \mathrm{bc}$ & $17.70 \mathrm{a}$ & $120.43 \mathrm{~b}$ & $210.91 \mathrm{a}$ & $146.97 \mathrm{a}$ \\
\hline
\end{tabular}

表 4 不同基因型烤烟各部位烟叶的钾含量 Table 4 Potassium content in different genotypes of flue-cured

\begin{tabular}{clll}
\multicolumn{4}{c}{ tobacco (\%) } \\
\hline $\begin{array}{c}\text { 株系 } \\
\text { Line }\end{array}$ & $\mathrm{X}_{2} \mathrm{~F}$ & $\mathrm{C}_{3} \mathrm{~F}$ & $\mathrm{~B}_{2} \mathrm{~F}$ \\
\hline $\mathrm{K} 326$ & $2.58 \mathrm{~b}$ & $2.42 \mathrm{c}$ & $1.91 \mathrm{c}$ \\
$\mathrm{K} 2$ & $2.83 \mathrm{a}$ & $2.65 \mathrm{a}$ & $2.64 \mathrm{a}$ \\
$\mathrm{K} 7$ & $2.74 \mathrm{a}$ & $2.77 \mathrm{a}$ & $2.67 \mathrm{a}$ \\
$\mathrm{K} 9$ & $2.45 \mathrm{~b}$ & $2.57 \mathrm{~b}$ & $2.00 \mathrm{~b}$ \\
\hline
\end{tabular}

条件下能进行正常代谢，随着外界养分供应浓度的增加， 养分利用效率也会逐渐下降, 贫痊环境中植物的养分利 用效率普遍较高, 但并非生长介质中养分有效性越高, 植物养分利用效率就越低 ${ }^{[26-27]}$, 与本文研究结论一致。这

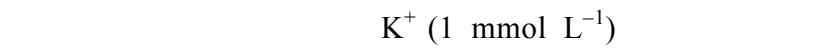
主, 是通过载体蛋白来完成, 该吸收机制被称为高亲和 吸收, 植物在高浓度 $\mathrm{K}^{+}\left(1 \sim 50 \mathrm{mmol} \mathrm{L}^{-1}\right)$ 时的吸收属 $\mathrm{K}^{+}$低 亲和吸收, 主要通过 $\mathrm{K}^{+}$通道完成 ${ }^{[28]}$ 。

针对本文的研究结果, 有几方面值得探讨。中国烟草 基因组计划实施以来，绘制完成线毛状烟草(Nicotiana tomentosiformis)和林烟草(Nicotiana sylvestris) 全基因组序 列图谱, 但未绘制完成红花烟草(Nicotiana tabacum)基因 组序列, 故本文分析的部分基因来自拟南芥。拟南芥和烟 草同作为转基因研究的模式植物，在拟南芥中鉴定过部 分基因与烟草及其他高等植物具有同源性, 并且, 也有 较多转拟南芥基因高含钾量烟草的报道, 本文所研究之 并非全部来自烟草, 但仍具有一定的参考意义。另外, 本 文所采用花粉管通道导入的DNA之方法, 于 20 世纪 80 年
代初期由我国学者周光宇提出, 是在植物授粉后向子房 注射含目的基因的DNA溶液, 利用植物在开花、受精过程 中形成的花粉管通道, 将外源DNA导入受精卵细胞, 并 进一步地被整合到受体细胞的基因组中，随着受精卵的 发育而成为带转基因的新个体, 作为一种常规育种方法 广为接受, 该方法导入外源DNA具有一定的偶然性。从分 子水平上看, 虽然就整个染色体基因组而言, 亲缘关系 较远的生物间的染色体和染色体外DNA的结构愈不亲和, 则愈互相排斥, 但从局部 DNA 片段来看, 两种植物的 部分结构却可能保持一定的亲和性。因而, 远缘 DNA 片 段在母本 DNA 复制过程中有可能被重组, 而使子代出 现变异。这种参与杂交的 DNA 片段可能带有可亲和远 缘物种的结构基因、调控基因、甚至是断裂的无意义的 DNA 片段。后两种DNA 片段如果整合到母本基因组中, 将同样可能影响母本基因表达而变异。本文采用花粉管通 道法导入空心莲子草、商陆和马齿苋的基因, 并以烟叶钾 含量指标高于 $2.5 \%$ 为主要选择压力进行多年系统选择, 虽然在导入时缺乏相应的分子证据作为支撑，但的确能 提高某些株系的烟叶钾含量，该方法仍具有实践指导性。

\section{References}

[1] Chaplin J R. Production factors affecting chemical compounds of the tobacco leaf. Recent Adv Tob Sci, 1980, (6): 3-63

[2] Sims J L, Casy M, Legget J E. Effect of transplant water fertilization on growth and chemical composition of burley tobacco. Annual report of the college of agriculture and the K. Y. Agric Exp Station, 1981, 59-60

[3] Cao Z-H(曹志洪), Hu G-S(胡国松). Relationship between con- 
trol of potassium and trace elements and quality of tobacco leaf. Soils (土壤), 1993, 25(3): 119-128 (in Chinese)

[4] Anderson J A, Huprikar S S, Kochian L V, Lucas W J, Gaber R F. Function expression of a probable Arabidopsis thaliana potassium channel in Saccharomycex cerevisiae. Proc Natl Acad Sci USA, 1992, 89: 3736-3740

[5] Sentenac H, Bonneaud N, Minet M. Cloning and expression in yeast of a plant potassium ion transport system. Science, 1992, 256: 663-665

[6] Lu L-M(鲁黎明). In silico cloning and bioinformatic analysis of TPK1 gene in tobacco. Sci Agric Sin (中国农业科学), 2011, 44(1): 28-35 (in Chinese with English abstract)

[7] Sano T, Becker D, Ivashikina N, Wegner L H, Zimmermann U, Roelfsema M R, Nagata T, Hedrich R. Plant cells must pass a $\mathrm{K}^{+}$ threshold to re-enter the cell cycle. Plant J, 2007, 50: 401-413

[8] Ache P, Becker D, Ivashikina N, Dietrich P, Roelfsema M R G, Hedrich R. GORK, a delayed outward rectifier expressed in guard cells of Arabidopsis thaliana, is a $\mathrm{K}^{+}$-selective, $\mathrm{K}^{+}$-sensing ion channel. FEBS, 2000, 486, 93-98

[9] Hosy E, Vavasseur A, Mouline K, Dreyer I, Gaymard F, Poree F, Boucherez J, Lebaudy A, Bouchez D, Very A A, Simonneau T, Thibaud J B, Sentenac H. The Arabidopsis outward $\mathrm{K}^{+}$channel GORK is involved in regulation of stomatal movements and plant transpiration. Proc Natl Acad Sci USA, 2003, 100: 5549-5554

[10] Xu J, Li H D, Chen L Q, Wang Y, Liu L L, He L, Wu W H. A protein kinase, interacting with two calcineurin B-like proteins, regulates $\mathrm{K}^{+}$transporter AKT1 in Arabidopsis. Cell, 2006, 125: 1347-1360

[11] Dai L-J(戴林建), Xu S-H(徐双红), Zhu L-S(朱列书), Zhong $\mathrm{J}$ (钟军), Xia $\mathrm{K}$ (夏凯). Studies on variation in characters of progeny obtained by introducing total DNA of high potassium plants to tobacco. Crop Res (作物研究), 2010, (2): 109-111 (in Chinese with English abstract)

[12] Dai L-J(戴林建)，Xu S-H(徐双红), Sun H-L(孙焕良), Wang $\mathrm{K}$ (王坤), Zhong J(钟军). SRAP analysis on the purity of tobacco D4 generation with portulaca DNA. Tob Sci (烟草科技), 2010, (7): 48-52 (in Chinese with English abstract)

[13] Yang T-Z(杨铁钊), Peng Y-F(彭玉富). Potassium accumulation characteristics of rich-potassium genotypic flue-cured tobacco. Plant Nutr Fert Sci (植物营养与肥料学报), 2006, 12(5): 750-753 (in Chinese)

[14] Yang T-Z(杨铁钊), Yang Z-X(杨志晓), Nie H-Z(聂红资), Zhang X-Q(张小全), Liu Y-J(刘友杰), Shang X-Y(尚晓颖), Ren Z-Y(任周营), Fan J-H(范进华). Potassium accumulation and root physiological characteristics of potassium-enriched fluecured tobacco genotypes. Acta Agron Sin (作物学报), 2009, 35(3): 535-540 (in Chinese with English abstract)
[15] Zhao X-Q(赵学强), Jie X-L(介晓否), Li Y-T(李有田), Xu X-J(许仙菊), Tan J-F(谭金芳), Hua D-L(化党领). Studies in screening indices and screening environments for efficient potassium wheat genotypes. Plant Nutr Fert Sci (植物营养与肥料学 报), 2006, 12(2): 277-281 (in Chinese with English abstract)

[16] Verwoerd T C, Dekker B M, Hoekema A. A small-scale procedure for the rapid isolation of plant RNAs. Nucl Acids Res, 1989, 17: 2362

[17] Guo Z-K(郭兆奎), Yang Q(杨谦), Yan P-Q(颜培强), Wan $\mathrm{X}-\mathrm{Q}$ (万秀清). Cloning and homology modeling of a potassium channel gene NKC1 from Nicotiana rustica. Acta Tab Sin (中国 烟草学报), 2008, 14(5): 63-68 (in Chinese with English abstract)

[18] Liu K, Luan S. Intracellular potassium sensing of SKOR, a shaker-type K-channel from Arabidopsis. Plant J, 2006, 46, 260-268

[19] Pilot G, Lacombe B, Gaymard F, Cherel I, Boucherez J, Thibaud J B, Sentenac H. Guard cell inward $\mathrm{K}^{+}$channel activity in Arabidopsis involves expression of the twin channel subunits KAT1 and KAT2. J Biol Chem, 2001, 276, 3215-3221

[20] Gaymard F, Pilot G, Lacombe B, Bouchez D, Bruneau D, Boucherez J, Michaux-Ferrière N, Thibaud J B, Sentenac H. Identification and disruption of a plant Shaker-like outward channel involved in $\mathrm{K}^{+}$release into the xylem sap. Cell, 1998, 94, $647-655$

[21] Mao D-R(毛达如). Plant Nutrition Research (植物营养研究). Beijing: Beijing Agricultural University Press, 1994. pp 132-135 (in Chinese)

[22] Min S-Z(闵水珠). Molecular biology research progress on plant potassium ion channel. Acta Agric Zhejiangensis (浙江农业学 报), 2005, 17(3): 163-169 (in Chinese with English abstract)

[23] Shin R, Schachtman D P. Hydrogen peroxide mediates plant root cell response to nutrient deprivation. Proc Natl Acad Sci USA, 2004, 101: 8827-8832

[24] Zhao X-Q(赵学强), Jie X-L(介晓否), Li Y-T(李有田), Xu X-J(许仙菊), Tan J-F(谭金芳), Hua D-L(化党领). Dynamics analysis of absorption of potassium ion in wheat with different genotypes. Plant Nutr Fert Sci (植物营养与肥料学报), 2006, 12(3): 307-312 (in Chinese with English abstract)

[25] Wang Z-Q(汪自强), Dong M-Y(董明远). Efficiency of using potassium for spring soybean varieties with different level of pitassium. Soybean Sci (大豆科学), 1996, 15(3): 202-207 (in Chinese with English abstract)

[26] Su B, Han X G, Huang J H, Qu C M. The nutrient use efficiency (NUE) of plants and its implications on the strategy of plant adaptation to nutrient stressed environments. Acta Ecol Sin, 2000, 20: $335-343$

[27] Bridgham S D, McClaugherty C A, Richardson C J, Pastor J. 
Nutrient-use-efficiency: a litter fall index, a model and a test along a nutrient availability gradient in North Carolina peat lands. Am Nat, 1995, 145: 1-21

[28] Jiang C-C(姜存仓), Wang Y-H(王运华), Lu J-W(鲁剑巍), Xu F-S(徐芳森), Gao X-Z(高祥照). Advances of study on the K-efficiency in different plant genotypes. J Huazhong Agric Univ
(华中农业大学学报)，2004，23(4)：483-487 (in Chinese with English abstract)

[29] Mpelasoka B S, Schachtman D P, Treeby M T, Thomas M R. A review of potassium nutrition in grapevines with special emphasis on berry accumulation. Aust J Grape Wine Res, 2003, 9: $154-168$

\section{科学出版社生物分社新书推介}

\section{《元基因组学：方法和步骤》（影印版）生命科学前沿}

出版时间：2012 年 3 月

书号：978-7-03-033858-7

定价: $¥ 80.00$

本书涵盖了元基因组文库的构建、元基因组文库的篮选技术和 针对不同活性目标产物的篮选方法, 并且提供了详细实用的实验操 作方法, 可以帮助希望从事元基因组学研究的科技人员尽快建立相 关的实验平台。

编辑推荐：该书由 Humana Press 出版，作者长期从事元基因组 学的研究, Rolf Daniel 还是国际权威期刊 Applied and Environmental Microbiology 的编委之一。

元基因组技术是目前研究微生物的一种热门技术, 以用于挖掘 新的功能基因（簇）资源，而这些资源可能应用于环境治理、工业 生物技术、新药开发等行业，其应用性非常广泛。本书偏重实用性， 包括了元基因组文库的构建，元基因组文库的篮选技术和针对不同 活性目标产物的篮选方法, 以期帮助从事元基因组学研究的科技人 员尽快建立相关的实验平台。

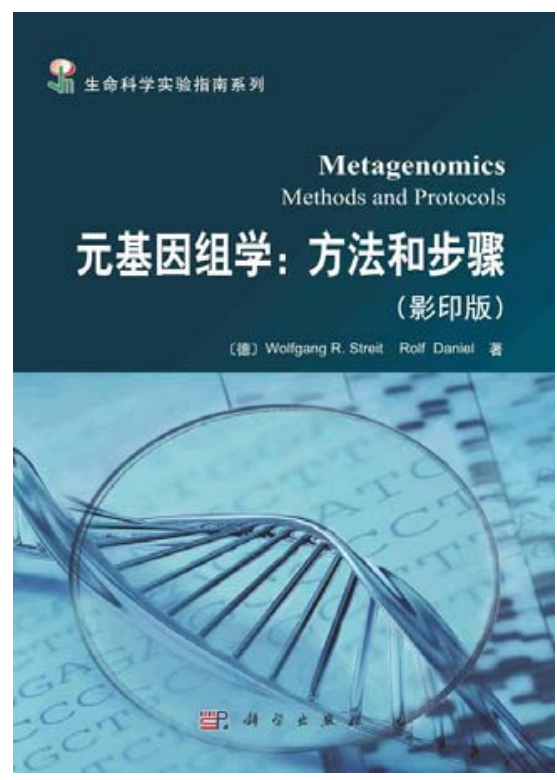

获取更多图书信息请您关注: http://www.lifescience.com.cn/ 欢迎各界人士邮购科学出版社各类图书

联系人: 科学出版社科学销售中心 周文宇 E-mail: zhouwenyu@mail.sciencep.com

联系电话: 010-64022646 010-64017321

网上订购: http://shop.sciencepress.cn 卓越网 当当网 京东图书 学士书店

更多精彩图书请登陆网站, 欢迎致电索要书目 